\title{
Replication of Saffran, Johnson, Aslin, \& Newport (1999) Statistical learning of tone sequences by human infants and adults, Exp. 2
}

\author{
Kara Murdock, Alyssa Savery, Kelsey Trimm, Juliani Vidal and Joshua K. Hartshorne \\ Department of Psychology, Boston College \\ Contact joshua.hartshorne@bc.edu
}

\begin{abstract}
We replicated Exp. 2 of Saffran, Johnson, Aslin, \& Newport (1999) Statistical learning of tone sequences by human infants and adults, Cognition, 70, 27-52, as part of a multi-year effort to replicate every adult statistical word segmentation study. While we were able to replicate the finding of above-chance statistical segmentation of tone sequences, many of the other findings reported in the original paper did not replicate.
\end{abstract}

Keywords: statistical learning, unsupervised learning, language acquisition, replication

In this paper, we describe a replication of Experiment 2 from a classic statistical word-segmentation paper by Saffran, Johnson, Aslin, and Newport (1999). This experiment was part of a larger project to systematically replicate as many experiments involving statistical word learning in adults as possible.

\section{Method}

\section{Subjects}

101 subjects were recruited through Amazon Mechanical Turk and paid for their participation. Subjects were randomly assigned to one of the two languages, with $51^{1}$ subjects in Language One and 50 in Language Two. We excluded twelve subjects who reported formal music activities (lessons, singing in a choir, etc.) in the last five years. We further excluded 17 who incorrectly answered any catch trials, leaving us with 72 subjects: 36 in Language One (mean age: 35, range: 23-59) and 36 in Language Two (mean age: 34, range: 21-59). All but one of these subjects were native speakers of English. All reported normal hearing.

\section{Known differences with original.}

- Our study had three times as many subjects (24 vs. 72) and thus considerably more power and precision.

- The original excluded subjects who had significant musical training since the 7 th grade. Given the wide age range in our subjects, this seemed to risk confound. Thus, we used the 5-year criterion instead.

\section{Possible differences with original.}

- The age range for the original subjects is unknown.

- The native languages of subjects in the original study are unknown.
- It is unknown how and whether subjects in the original study were compensated (course credit, money, etc.).

\section{Materials}

Tone sequences were constructed using free online services; first it was necessary to pinpoint the exact frequencies of the tones in hertz using an Online Tone Generator (synzalski.com), and then the tones were created and downloaded using a Sine Tone Generator (audiocheck.net).

Tones were constructed out of eleven pure tones of the same octave (starting at middle $\mathrm{C}$ within a chromatic set) and were the same length $(.33 \mathrm{~s})$. In order to form six tone words, the pure tones were combined into groups of three. Language One consisted of: ADB, DFE, GG\#A, FCF\#, D\#ED, and $\mathrm{CC \# D}$. Some tones appeared in only one word $(\mathrm{G \#}$ for example) while others occurred in multiple words (D for example). The six tone words were then used to create six different blocks that each contained 18 words. The tone words were concatenated in a random order without silence between words, and tone words did not occur twice in a row. Each word was concatenated together 70 times in order to produce a seven-minute continuous stream of tones, which was called Language One. A given tone stream, for example, would be DFEFCF\#CC\#DD\#EDGG\#A. As in the Saffran et al. study, there were no acoustic markers of word boundaries. The average transitional probability between tones within words of Language One was 0.64 (range: 0.42 - 1.00). In contrast, average transitional probability between tones spanning word boundaries was 0.18 (range: 0.04 to $0.59)$.

Saffran et al. (1999) constructed two more languages. One of them (Language Two) was used for their Experiment 1 and is not discussed further here. The third language used

\footnotetext{
${ }^{1}$ We intended to recruit 50 for each, but ended up one over due to experimenter error.
} 
the same set of 11 tones, such that its words were part-words with respect to Language One, while words from Language One were part-words with respect to Language Three. The transitional probabilities between the new tone and the two tones taken from a word were always zero with respect to the trained language. To generate a part-word, either the first or third tone was substituted with a different tone (e.g. G\#DB). Three part-words contained the first two tones of words plus a new third tone, and three contained the final two tones of words plus a new first tone. The tone words for the new Language Three were G\#DB, DFF\#, FG\#A, C\#CF\#, D\#EG\#, and $\mathrm{CC \# B}$. The average within-word transition probability was $0.56(0.42-1.00)$. The average transition probability between words in Language Three was 0.18 (0.05 - 0.40).

During the test phase, subjects were required to discriminate trained words from foils. The six trained words from one language were paired exhaustively with six words from the untrained language, forming 36 test trials. Because these foils shared some structure with the trained words, this was expected to be relatively difficult. We also added six catch trials that compared a real word against something that was obviously not in the training in order to find which subjects were not paying attention. They were always the last six items in the test. The tone sequences of each trial were separated by a 0.75 second interval of silence, and the trials themselves were separated by 1 second.

\section{Known differences with original.}

- Saffran et al. (1999) generated stimuli using SoundEdit 16. We used audiocheck.net.

- Saffran et al. (1999) used a 5 second delay between each test trial. We used a shorter, 1 second delay because we did not want subjects to think the webpage was not loading.

- Saffran et al. (1999) generated two different random orders of the test trials. In contrast, we randomized the order of questions for each subject in order to help eliminate any effects of order.

- We have slightly different within-word and betweenword transition probabilities from what they report, likely due to differences in randomization.

- The original provided subjects with three practice trials at the start of the test, which we did not. ${ }^{2}$

\section{Apparatus}

Participants were recruited using Amazon Mechanical Turk through the Boston College Language Learning Lab and used their own computers and listening devices in order to listen to the tone streams and complete the experiment. The experiment was programmed using jsPsych (de Leeuw, 2014) and run on Amazon Mechanical Turk using PsiTurk Gureckis et al. (2016).
Known differences with original. The original experiment was conducted in an IAC sound-attenuated booth. Stimuli were presented using an Aiwa tape deck and a Proton speaker.

\section{Procedure}

Subjects were run independently and individually. Firstly, subjects were instructed that they would hear a recording of continuous tones. However, they were not told which aspects of the tone sequence would be tested. They were told to relax and try not to analyze the sound stream. Subjects then listened to the seven-minute-long recording of one of the two tone streams (Language One or Language Three) described above, repeated three times. Each of the three sevenminute listening sessions was followed by a short, subjectpaced break. After a total of twenty-one minutes of listening, subjects were exposed to the 36-question test. They were instructed to indicate the most familiar tone sequence on each test trial. The correct choice for subjects exposed to Language One was the incorrect choice for subjects exposed to Language Three. The order of the test trials was randomized for each subject.

After the primary test trials, all subjects answered the 6 catch trials. The order was randomized for each subject. The catch trials followed the primary test trials immediately, without any overt distinction.

\section{Known differences with original.}

- The original study did not include catch trials. Note that because the catch trials follow the primary test trials, they cannot affect the results of those trials (except in that we can use the catch trials to distinguish poor learners from noncompliant subjects).

- In the original, subjects provided written responses.

- It is not clear how long the breaks between blocks were in the original, or whether these were subject-paced.

\section{Results}

Original Analyses. In Language One, the mean score for subjects was $22.1 \quad(\mathrm{SE}=0.9)$ correct out of 36 total questions $(61 \%)$, which was significantly above chance $\left(\mathrm{t}(35)=4.5, \mathrm{p}=7.4^{*} 10^{-5}\right)$. Performance was above chance for all the trained words except FCF\# (ps<.05). In contrast, Saffran et al. (1999) report above-chance performance for only four of the six trained words.

Language Three had a mean of 22.3 ( $\mathrm{SE}=0.8)$ correct answers $(62 \%)$, which was likewise above chance $(\mathrm{t}(35)=5.7$, $\left.\mathrm{p}=1.8 * 10^{-6}\right)$. All but one trained word $(\mathrm{CC} \# \mathrm{~B})$ were learned

\footnotetext{
${ }^{2}$ The practice trials are not described in the original, making them difficult to reproduce and their exact purpose unclear.
} 
at above-chance levels (ps<.05), whereas subjects in the original were above chance on four of six.

Performance was not significantly different across the two languages by subjects $(\mathrm{t}(70)=0.2, \mathrm{p}=.83)$ or by items $(\mathrm{t}(10)=0.1, \mathrm{p}=.89)$, consistent with what was reported by Saffran et al. (1999).

Saffran et al. (1999) found that accuracy was lower in their Exp. 2 than in their Exp. 1. Since we also replicated their Exp. 1 (Murdock et al., 2017), we were able to carry out the same analysis, with the same results: overall accuracy was lower in Exp. $2(\mathrm{M}=62 \%, \mathrm{SE}=2 \%)$ than in Exp. 1 $(\mathrm{M}=67 \%, \mathrm{SE}=2 \% ; \mathrm{t}(147)=2.4, \mathrm{p}=.02)$.

Saffran et al. (1999) found that accuracy was better for foils where the first tone had been changed relative to foils where the last tone had been changed. We likewise found a slight advantage for foils where the first tone had been changed $(63 \%, \mathrm{SE}=2 \%)$ compared with foils where the last tone had been changed $(61 \%, \mathrm{SE}=2 \%)$. However, this did not rise to the level of significance, despite our much larger sample size $(\mathrm{t}(71)=0.8, \mathrm{p}=.41)$.

Saffran et al. (1999) also compared their results to Saffran et al. (1996) Exp. 2. Since we have not yet replicated that experiment, we were unable to carry out this comparison. The authors also reported a number of analyses comparing items with different melodic structure. We did not attempt to reproduce these analyses.

Revised Analyses. There are two important limitations to the original analyses conducted by Saffran et al. (1999). First, their use of t-tests on accuracy data violates core assumptions of t-tests: that the data are continuous and error is normally distributed. Violation of these assumptions can lead both to false positives and false negatives (Jaeger, 2008). Binomial models are more appropriate (Jaeger, 2008). ${ }^{3}$

Additionally, they treat items as fixed effects. Thus, any conclusions are specific to the stimuli used. No assessment of generalization can be made. Statisticians have suggested two options. The first is to conduct analyses separately over subject averages (as Saffran et al. (1999) did) and also over item averages (Clark, 1973). The second option is to use mixed effects models (Baayen et al., 2008).

Given these considerations, we re-analyzed the data using binomial mixed effects models, implemented in R using the lme4 package, ver. 1.1-12 (Bates et al., 2017). We considered main effects of language and foil type (changedfirst/changed-last), as well as their interaction. We set the contrasts to center the variables, such that the intercept is equal to the grand mean. We considered two possible random effects structures.

The first estimated random intercepts for each word/foil pair and for each subject, as well as a random slope of foil type for each subject. The second estimated random intercepts for each trained word and foil separately, random intercepts for each subject, and a random slope of foil type for each subject. ${ }^{4}$ The former provided a better fit as assessed by conditional AIC (6314 v. 6360). (We calculated cAIC using Stein formulas, as implemented in Greven \& Kneib (2010) and Saefken \& Ruegamer (2015)). Though this should be taken with some caution since the conditional AIC failed to converge for the latter model. However, the results of the two models were similar. Thus, we use the former random effects structure throughout, including random slopes where appropriate.

We found a significant intercept $(0.51, \mathrm{SE}=0.08$; Wald's $\mathrm{z}$ $\left.=6.0, \mathrm{p}=1.6^{*} 10^{-9}\right)$, indicating overall above-chance learning. ${ }^{5}$ The main effect of language was not significant $(\mathrm{B}=$ 0.02, $\mathrm{SE}=.07$; Wald's $\mathrm{z}=0.20, \mathrm{p}=.84$ ), nor was the main effect of foil type $(\mathrm{B}=-0.07, \mathrm{SE}=0.06$; Wald's $\mathrm{z}=1.3$, $\mathrm{p}=.19)$. The interaction was likewise not significant $(\mathrm{B}=$ $0.09, \mathrm{SE}=0.6$; Wald's $\mathrm{z}=1.5, \mathrm{p}=.15$ ).

We followed these analyses with binomial mixed effects models for each trained word individually, with a random intercept of foil. Exactly half of the trained words exhibited above-chance discrimination ( $\mathrm{ps}<.05)$.

Thus, the mixed effects regression analyses largely supported the findings from the previous section based on t-tests.

Saffran et al. (1999) found that accuracy was lower in their Exp. 2 than in their Exp. 1. Since we also replicated their Exp. 1 (Murdock et al., 2017), we were able to carry out these analysese with a binomial mixed effects model with random intercepts of subject and file. The main effect of experiment was significant $(\mathrm{B}=0.28, \mathrm{SE}=0.13$; Wald's $\mathrm{z}=2.2$, $\mathrm{p}=.03)$. Thus, we replicated lower performance in Exp. 2 $(\mathrm{M}=62 \%, \mathrm{SE}=2 \%)$ than in Exp. $1(\mathrm{M}=67 \%, \mathrm{SE}=2 \%)$.

\section{Discussion}

We found mixed support for the conclusions of Saffran et al. (1999) Exp. 2. Like them, we found successful discrimination of trained tone "words" from foils that differed from trained words on a single tone. Like them, we found that success on this task was significantly different from discriminating trained words from foils that did not contain any transitions in common with the trained words.

However, we were unable to replicate their finding of better discrimination of trained words from part-word foils that differed from trained words on the first tone as opposed to the last tone. We did not attempt to replicate their analyses of the effect of tonal structure on learning.

Note that we used a strict criterion for replication: significant results in the same direction. This is both for simplicity

\footnotetext{
${ }^{3} \mathrm{An}$ intermediate option is to transform accuracy measures to log-odds, though this method is less accurate than a binomial model.

${ }^{4}$ While a random slope of foil type with respect to trained word was theoretically possible, such a model failed to converge.

${ }^{5}$ Note that because of the contrast structure, these parameter estimates are half of the estimated effect size.
} 
and because Saffran et al. (1999) do not provide the information needed for more sophisticated measures of replication (e.g., standard errors).

As with any replication, our replication was inexact. We used different foils, and it may be that ours were a priori more difficult to distinguish from the words. Second, it is likely that our population differed from the original along a number of demographic dimensions. Finally, the fact that our subjects were tested over the Internet could result in them being less attentive. While we attempted to address this by using an attentional screen, we cannot completely rule out this possibility. Whether less attention could explain our failure to replicate the foil-type manipulation is unclear.

\section{Data and Code}

Data, analysis code, and experiment code are available at osf.io/5d794.

\section{References}

Baayen, R. H., Davidson, D. J., \& Bates, D. M. (2008). Mixedeffects modeling with crossed random effects for subjects and items. Journal of Memory and Language, 59(4), 390-412.

Bates, D., Maeschler, M., Bolker, B., Walker, S., Christensen, R. H. B., Singmann, H., .. Green, P. (2017). Linear mixed-effects models using 'eigen' and s4. cran.rproject.org/web/packages/lme4/index.html.

Clark, H. H. (1973). The language-as-fixed-effect fallacy: A critique of language statistics in psychological research. Journal of Verbal Learning and Verbal Behavior, 12(4), 335-359. de Leeuw, J. R. (2014). jspsych: A javascript library for creating behavioral experiments in a web browser. Behavior Research Methods, 47(1), 1-12.

Greven, S., \& Kneib, T. (2010). On the behaviour of marginal and conditional aic in linear mixed models. Biometrika, 773-789.

Gureckis, T. M., Martin, J., McDonnell, J., Rich, A. S., Markant, D., Coenen, A., ... Chan, P. (2016). psiTurk: An open-source framework for conducting replicable behavioral experiments online. Behavior Research Methods, 48(3), 829-842.

Jaeger, T. F. (2008). Categorical data analysis: Away from ANOVAs (transformation or not) and towards logit mixed models. Journal of Memory and Language, 59(4), 434-446. doi: 10.1016/j.jml.2007.11.007

Murdock, K. N., Savery, A. A., Trimm, K. A., Vidal, J., \& Hartshorne, J. K. (2017). Replication of saffran, johnson, aslin, \& newport (1999) statistical learning of tone sequences by human infants and adults. doi: 10.17605/OSF.IO/QMPTZ

Saefken, B., \& Ruegamer, D. (2015). Conditional akaike information criterion for lme4. cran.r-project.org/web/packages/cAIC4/.

Saffran, J. R., Johnson, E. K., Aslin, R. N., \& Newport, E. L. (1999, Feb). Statistical learning of tone sequences by human infants and adults. Cognition, 70(1), 27-52. 